\title{
THE CAROTID PULSE II: RELATION OF EXTERNAL RECORDINGS TO CAROTID, AORTIC, AND BRACHIAL PULSES
}

\author{
BY \\ BRIAN ROBINSON \\ From St. George's Hospital, London S.W.1 \\ Received May 10, 1962
}

As it passes from the proximal aorta to the periphery, the normal pulse undergoes striking changes, mainly due to peripheral reflections, which result in it becoming sharper and increased in amplitude (Kroeker and Wood, 1955; McDonald and Taylor, 1959). The abnormal pulse is also subject to change, and alterations in the central pulse may, as a result, become either more or less obvious in the periphery. Thus the characteristic pulse of aortic regurgitation is made more obvious by transmission (Wright and Wood, 1958). The typical central pulse of aortic stenosis, on the other hand, may be so altered by transmission that the brachial fails to show a diagnostic pattern (Hancock and Fleming, 1960). As a result of the inadequacy of the brachial pulse in diagnosis, the carotid pulse, recorded by external means, is now increasingly used as a guide to central events (Duchosal et al., 1956; Daoud, Reppert, and Butterworth, 1959; Robinson, 1963). The present study is an attempt to assess how closely external carotid tracings reflect the central aortic pulse and in what ways they are superior to brachial pulse recordings. The relation of external carotid tracings to simultaneous carotid pressure recordings will first be established, and various types of external carotid will then be compared with the aortic and brachial pulse.

\section{METHOD}

The carotid pulse was recorded externally by a method similar to that of Duchosal et al. (1956) using an air-filled neck cuff (Robinson, 1963). When the carotid pressure pulse was to be recorded at the same time, a mercury and rubber strain gauge was used, having two loops around the neck between which the carotid artery was punctured with a fine needle. Aortic pulse recordings (through a catheter) and brachial pulse recordings (through a needle) were obtained during routine cardiac catheterization.

Simultaneous recordings of the external and pressure pulses in the carotid were made in 6 patients: 4 had aortic stenosis with varying degrees of regurgitation, one had pure aortic regurgitation, and one hypertension. External carotid and aortic pressure pulses were obtained in 43 patients: for technical reasons the recordings were made on separate occasions. Cardiac abnormalities other than aortic valve disease were present in 11 patients, and included mitral regurgitation, Fallot's tetralogy, and ischæmic heart disease; 8 patients had pure aortic regurgitation ( 4 with additional mitral valve disease), and 2 had patent ductus arteriosus; and 23 had aortic stenosis with or without regurgitation.

External carotid and direct brachial pressure pulses were obtained in 23 patients, the recordings being made at different times: in 2 patients the carotid pressure pulse was recorded for comparison with the brachial. Aortic stenosis was present in 10 patients, while 15 had disorders not involving the aortic valve.

Terminology. The brachial and aortic pulses commonly show two inflections analogous to those seen in the carotid, and the measurements and descriptive terms used in this paper are those previously defined in relation to the carotid pulse (Robinson, 1963).

\section{RESULTS}

Relation of External Carotid to Carotid $F$ issure Pulse. In all 5 patients with aortic valve disease, the external pulse followed the pressure pulse closely throughout systole (Fig. 1, A, B, and C); in 
diastole, however, perhaps due to a venous artefact, the external pulse tended to fall less rapidly than the pressure pulse and was sometimes convex upwards. In the patient with hypertension the external pulse differed from the pressure pulse in the later part of systole, having a relatively flattened appearance (Fig. $1 \mathrm{D}$ ). In every case, however, the external recording gave the correct timing of the major events in the pulse wave such as the summit and the incisura.
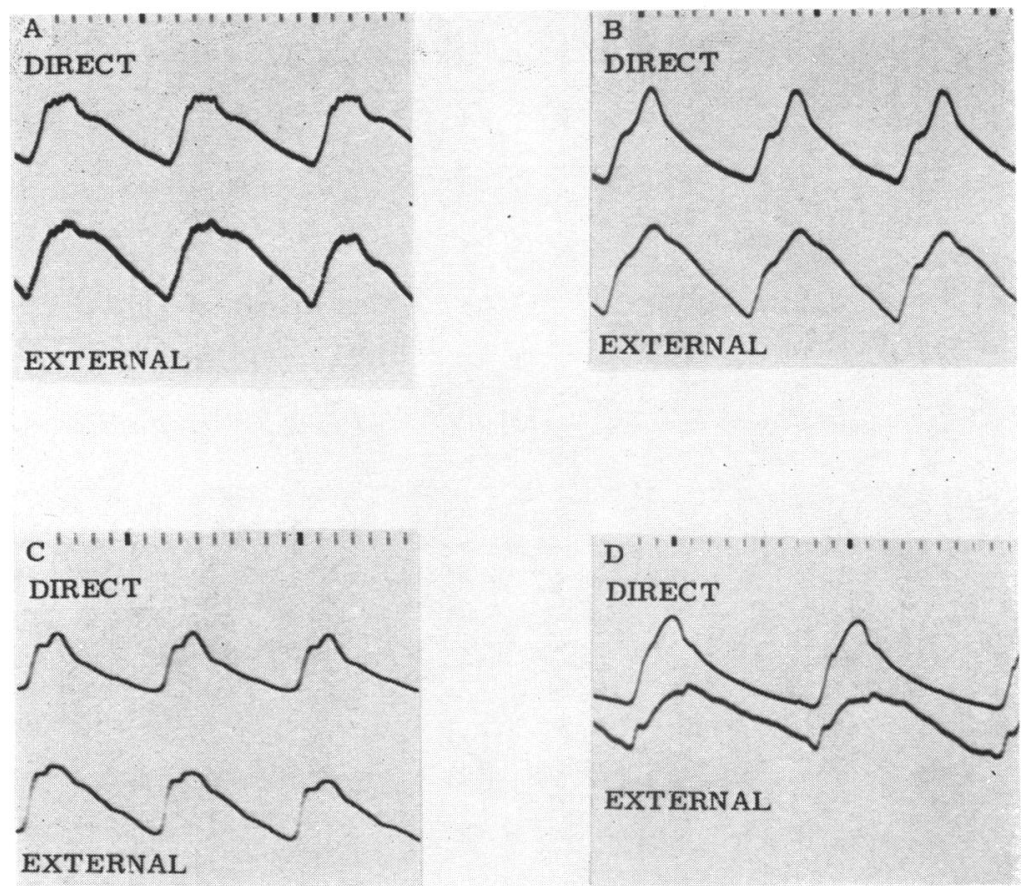

FIG. 1.-Simultaneous recordings of direct carotid pressure pulse and external carotid pulse. (Time intervals, 0.1 and 1.0 sec.) (A) Aortic stenosis; (B) aortic stenosis and regurgitation; (C) aortic regurgitation; (D) hypertension. In the absence of hypertension, the external recording satisfactorily reproduces the systolic part of the pressure pulse.

Relation of External Carotid to Aortic Pressure Pulse. The degree to which the external carotid differed from the aortic pulse varied considerably from case to case, but the ways in which it differed were almost always the same. The initial rise became steeper and taller while the higher frequency components, such as the incisura and systolic vibrations, were attenuated. These changes were seen in varying degree in the 11 patients without aortic valve disease (Fig. 2 A and B), but the carotid usually resembled the aortic pulse quite closely. In 7 cases the upstroke time in the carotid was similar to that in the aorta but in the other 4 it was shorter by $0.03-0.08 \mathrm{sec}$. The carotid ejection time was either equal to that in the aorta (allowance being made for differences in heart rate) or shorter by up to $0.03 \mathrm{sec}$. This agrees with the finding of Weissler, Peeler, and Roehll (1961) that the ejection time in the external carotid is equal to, or slightly shorter than, that in simultaneous aortic recordings.

Most of the patients with aortic regurgitation showed differences between the carotid and aortic pulses, which were moderate in degree, but two who had severe regurgitation showed an unusually great increase in the steepness and height of the initial rise in the carotid (Fig. 3A), and this was also seen in two patients with a large patent ductus arteriosus. The upstroke time in the carotid was 

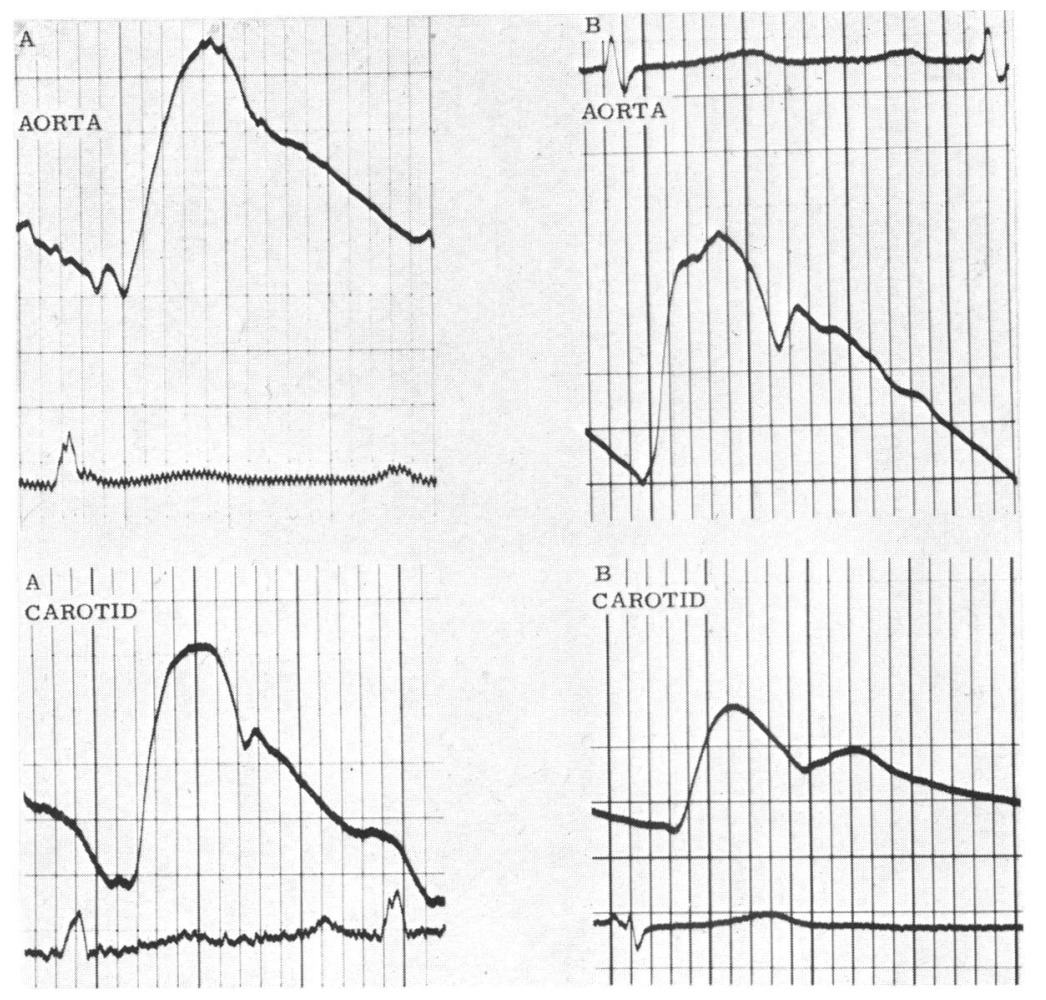

FIG. 2.-Comparison of aortic pressure pulse and external carotid. (A) Fallot's tetralogy; the initial rise is a little steeper in the carotid but the tracings are otherwise similar. (B) mitral regurgitation; the initial rise is relatively taller in the carotid so that the summit is $0.04 \mathrm{sec}$. earlier than in the aorta. In this and subsequent illustrations, the pulses cannot be compared with respect to height as the amplification has not been kept constant: the tracings are not simultaneous.

considerably reduced in one patient by the increase in the initial rise, but the carotid ejection time in this group, as in the previous one, was either the same as that in the aorta or slightly shorter.

In 17 of the 23 patients with aortic stenosis, the carotid pulse, although showing some alterations, had the same general contour as the aortic pulse and retained all the important features (Fig. 4A). In three patients, all of whom had severe regurgitation in addition to stenosis, the carotid showed a much taller initial rapid rise than the aortic pulse, but the summit was unaffected so the upstroke time was not greatly changed (Fig. 3B). In another three patients, with relatively pure aortic stenosis and typical changes in the aortic pulse, the carotid upstroke was altered so as to form a new earlier summit and the upstroke time was reduced by $0 \cdot 10-0 \cdot 12 \mathrm{sec}$. (Fig. 4B). Even when these patients with gross shortening are excluded, the carotid upstroke time did not correlate well with that in the aorta, being from $0.06 \mathrm{sec}$. longer to $0.07 \mathrm{sec}$. shorter (mean of $0.02 \mathrm{sec}$. shorter). The carotid ejection time in most of the patients with aortic stenosis was not more than $0.03 \mathrm{sec}$. above or below that in the aorta, but in four it was shorter by $0.04-0.06 \mathrm{sec}$. This range of differences is greater than in patients without aortic stenosis, and the reason for this is not clear. Systolic vibrations, present in the aortic pulse in 21 of the 23 patients, were often attenuated in the carotid, but in only one patient were they lost.

In summary, the external carotid tracing usually showed the main features of the aortic pressure pulse, but sometimes, particularly when there was a large stroke volume, the initial rise was con- 

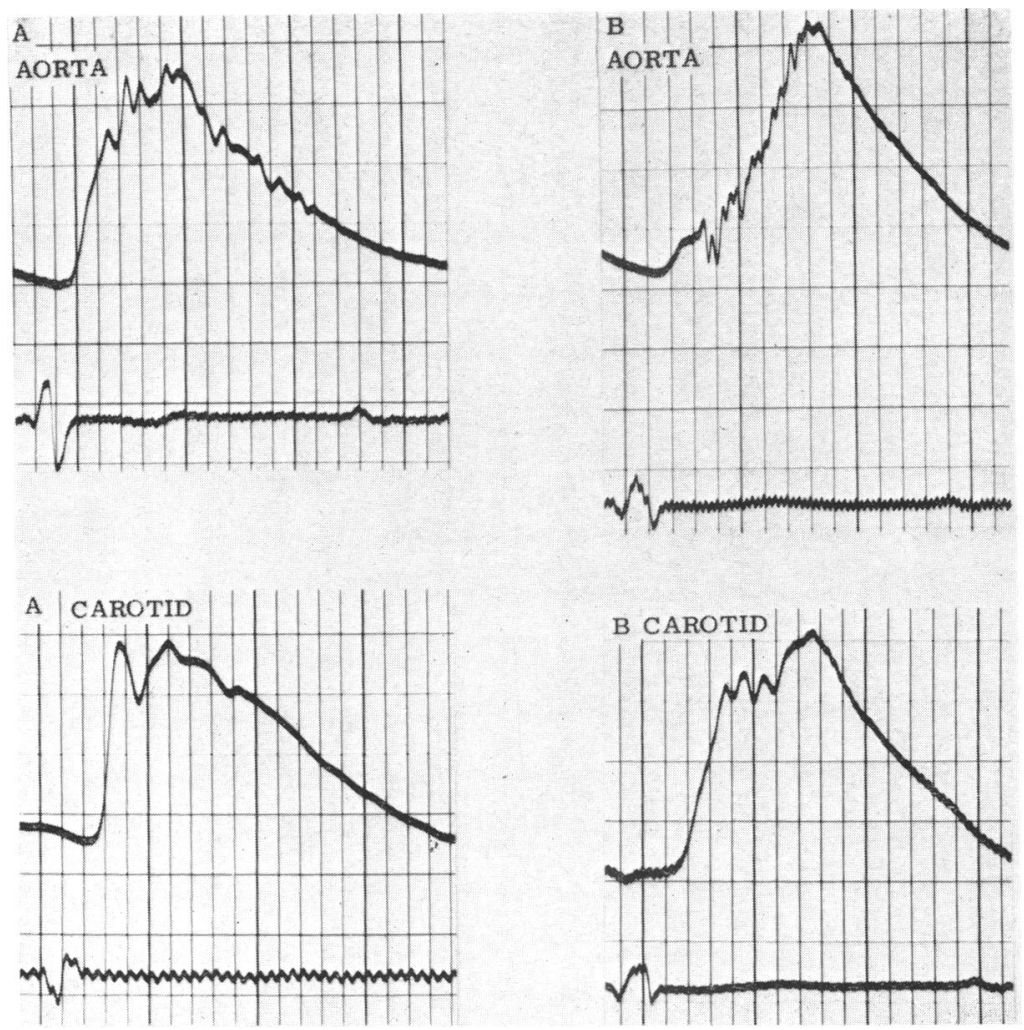

Fig. 3.-Comparison of aortic pressure pulse and external carotid. (Time intervals, 0.04 and $0.20 \mathrm{sec}$.) (A) Pure aortic regurgitation. The initial rise in the carotid is sharper and taller than in the aorta. (B) Mild aortic stenosis with moderate regurgitation. The initial rapid rise is much taller in the carotid than in the aorta.

siderably augmented; and higher frequency features were attenuated but seldom lost. Withdrawal of the aortic catheter to the innominate or subclavian always resulted in the pulse changing so that it became more like the carotid: this confirms that the differences which have been described are due to transmission, and are not merely the result of making the recordings at different times.

Relation of External Carotid to Brachial Pressure Pulse. The transmission changes seen in the brachial pulse were a development of those seen in the carotid: the rapid initial rise usually showed a further increase in size, although occasionally it was lost if it was small in the carotid; and higher frequency features were attenuated and systolic vibrations were usually lost. The normal carotid pulse was associated with a brachial pulse in which the initial rise was augmented so that the summit was formed by the first inflection, whether or not this had formed the summit in the carotid (Fig. 5A). As a result, the normal brachial upstroke time was always short and in 7 cases fell between 0.08 and $0.12 \mathrm{sec}$. although the carotid upstroke time in the same cases had varied widely. When the initial rapid rise in the carotid was less tall than normal (as for example in hypertension), it sometimes failed, despite augmentation, to give rise to the normal early summit in the brachial (Fig. 6A). The brachial summit then corresponded to the second inflection in the carotid, and the brachial upstroke time could in this way be prolonged in the absence of aortic stenosis. The incisura in the brachial was usually attenuated, often to such an extent that it was difficult to measure the ejection time accurately. 

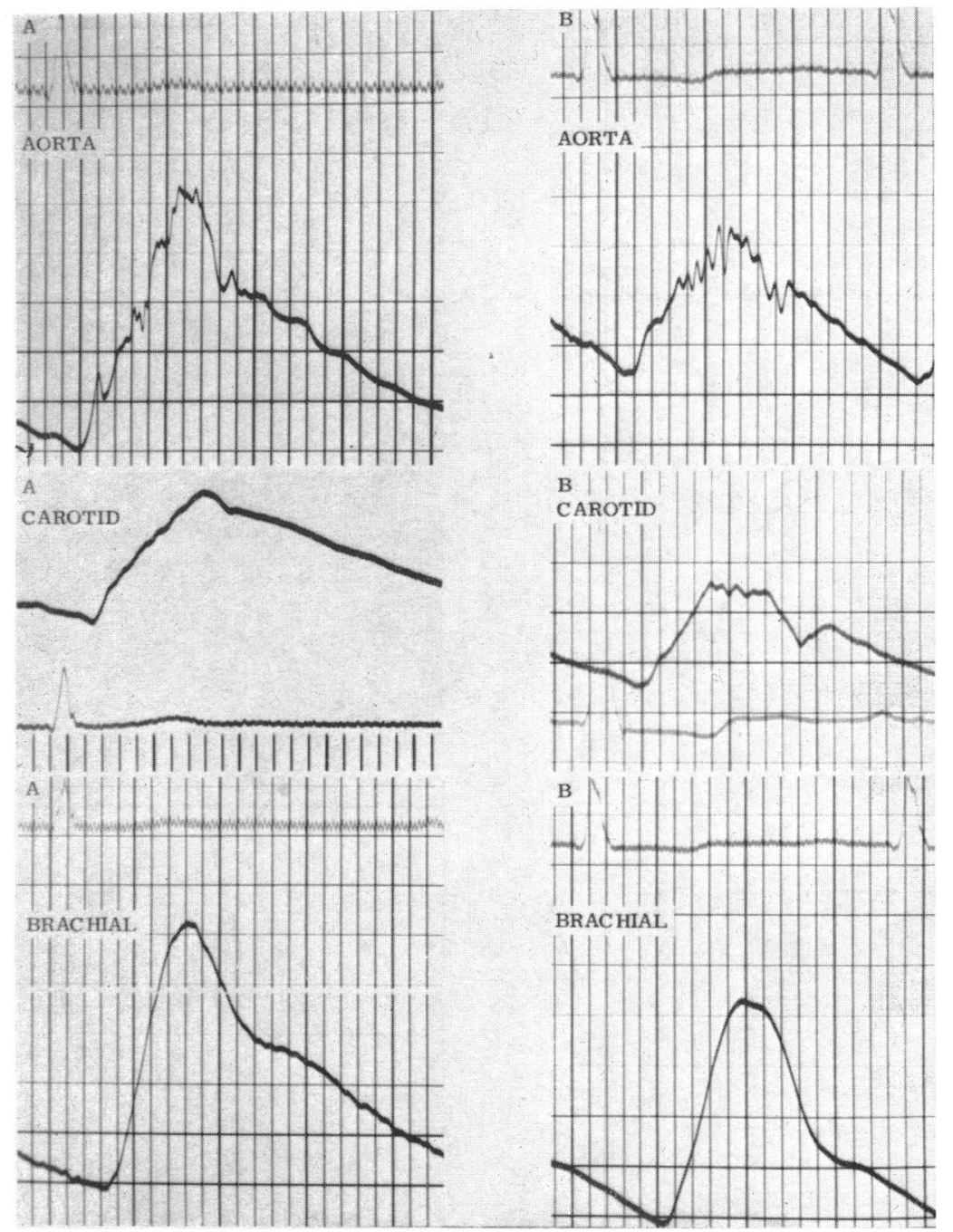

FIG. 4.-Comparison of aortic pressure pulse, external carotid, and brachial pressure pulse (time intervals, 0.04 and $\mathbf{0 . 2 0} \mathrm{sec}$.). (A) Mild aortic stenosis. The external carotid reproduces the small initial rapid rise, slow upstroke. systolic vibrations, and incisura of the aortic pulse. The brachial pulse has a slow upstroke but the other features are lost. (B) Subvalvular aortic stenosis. The external carotid shows some change compared with the aortic pulse and the summit is earlier, but the small initial rapid rise, systolic vibrations, and incisura are preserved. The brachial pulse has a slow upstroke but is otherwise featureless.

Of the patients with aortic stenosis, 6 who had a small or absent rapid rise in the carotid showed a featureless brachial pulse with a smooth rise to the summit (Fig. $4 \mathrm{~A}$ and B): with one exception, the upstroke time in the brachial was shorter than in the carotid, the difference varying from 0.06$0.11 \mathrm{sec}$. In four patients who had significant regurgitation in addition to stenosis, the carotid pulse showed a rapid initial rise of up to half its total height and in each case the rapid rise was not only present but augmented in the brachial. In one patient, a rapid rise in the carotid of only moderate height was associated with a typical bisferiens brachial pulse (Fig. 6B): in another with a 


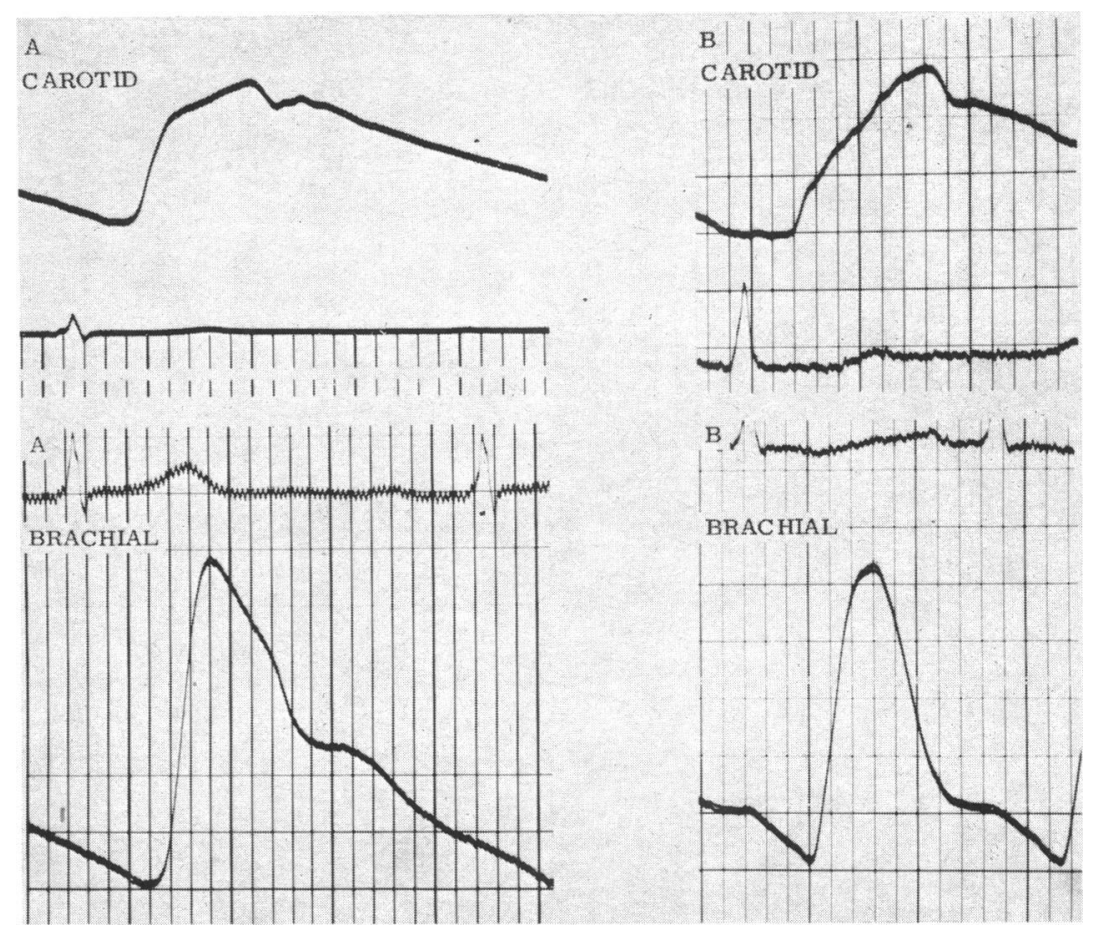

FIG. 5.-Comparison of external carotid and brachial pressure pulse. (Time intervals, 0.04 and $0.20 \mathrm{sec}$.) (A) Normal. The initial rise is relatively taller in the brachial so that the summit is formed by the first inflection and not as in the carotid, by the second. (B) Moderate aortic stenosis. The external carotid shows diagnostic changes with small initial rapid rise, slow upstroke and systolic vibrations: the brachial pulse is normal.

smaller rise in the carotid, a well-marked anacrotic notch was seen in the brachial. Systolic vibrations were seen in the carotid pulse in 7 patients, but were transmitted to the brachial in only one. The incisura was nearly always lost so that the ejection time was difficult to measure. As a result of these transmission changes the diagnosis of aortic stenosis could be made with confidence from the brachial pulse in only 7 patients, although the carotid had shown typical changes in all 10 . In one patient with an external carotid pulse characteristic of aortic stenosis, augmentation of the initial rise and loss of systolic vibrations resulted in a normal brachial pulse (Fig. 5B).

In summary, the brachial pulse often shows considerable differences from the external carotid, and in aortic stenosis this may result in the loss of important diagnostic features. When there is coexistent aortic regurgitation, however, the rapid initial rise in the carotid may be not only preserved but augmented in the brachial.

\section{Discussion}

The close relation found between the carotid pressure pulse and external recordings would be expected, since the arterial wall, although strictly a visco-elastic structure, shows an approximately linear relation between pressure and volume at the frequencies involved (Peterson, Jensen, and Parnell, 1960). The deviation seen in the hypertensive patient is presumably due to the pressure exceeding the elastic range, since the artery would then become less distensible so that the external pulse would be relatively flattened.

The changes in the pulse resulting from transmission have been analysed by McDonald and Taylor 


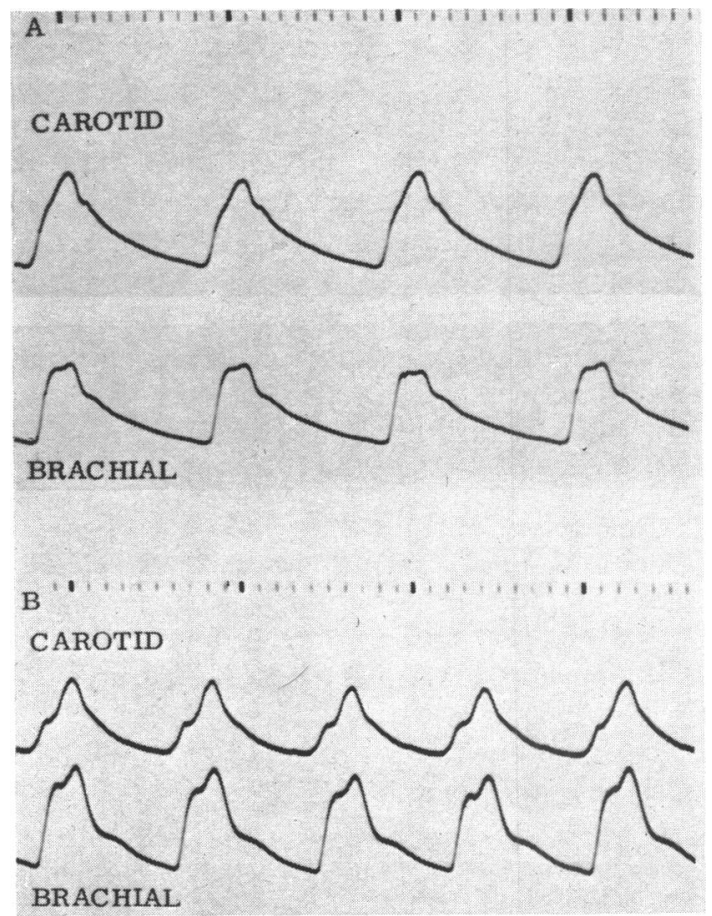

FIG. 6.-Comparison of carotid and brachial pressure pulse. (Time intervals, 0.1 and 1.0 sec.) (A) Hypertension; the initial rapid rise in the carotid is of moderate height, but although augmented in the brachial does not become tall enough to form a new, earlier summit. (B) Aortic stenosis and regurgitation; the initial rapid rise in the carotid halts abruptly and is followed by a short plateau; in the simultaneously recorded brachial, the initial rapid rise is augmented so that a typical bisferiens pulse results.

(1959) who have shown that distortion and increase in amplitude are due to peripheral reflections which augment to a varying extent components of different frequency in the pulse wave. Selective amplification of the frequencies responsible for the initial rapid rise explains the almost invariable increase in the size of this feature which occurs on transmission of both normal and abnormal pulses. It is important to realize that primary and reflected waves are completely fused and the reflected component cannot be distinguished as a separate entity during systole: the second inflection ("tidal wave") is not the reflection of the first inflection ("percussion wave"). Another important influence on the form of the pulse is the damping effect of the arterial wall. This causes attenuation of transmitted waves, which is greater in degree the higher the frequency of the disturbance.

As the pulse proceeds outwards, the variable peripheral factors that cause transmission changes become progressively more important in determining its form; and, in consequence, it becomes progressively less satisfactory as a guide to cardiac abnormalities. It is therefore not surprising that the brachial pulse is quite often within normal limits in aortic stenosis even though the aortic pulse is almost always abnormal (Hancock and Fleming, 1960). Transmission of the pulse brings this about in two ways. First, attenuation of systolic vibrations removes a diagnostic feature that is of great help in the central pulse. Secondly, distortion of the wave form, because of its variability, increases the overlap between stenotic and non-stenotic pulses making it more difficult to distinguish between them. For example, the upstroke time in the carotid pulse of 10 patients with aortic stenosis ranged from $0.01-0.29 \mathrm{sec}$., and was above the normal maximum $(0.26 \mathrm{sec}$.) in eight: the upstroke time in the brachial pulse of the same patients had a similar range $(0 \cdot 12-0.28 \mathrm{sec}$.) but transmission had so altered the distribution within the range that the normal maximum $(0.21 \mathrm{sec}$.) was exceeded in only 
three. Distortion of the pulse wave does not, however, invariably lead to loss of information. The rapid initial rise seen in the central pulse when aortic stenosis and regurgitation are combined is often augmented in transmission so that it is more obvious in the brachial pulse than in the carotid.

The carotid is influenced by transmission changes to a much lesser extent than the brachial, and usually retains the essential features of the aortic pulse. Even when its contour shows important differences from that of the aortic pulse, as in certain cases of aortic valve disease, recognition of the cardiac lesion is not made any more difficult, and, indeed, external carotid recordings have been found a reliable means of detecting the presence of aortic stenosis (Robinson, 1963). The carotid is thus a good guide to the information contained in the aortic pulse, much of which is lost in transmission to the brachial. Routine examination of the cardiovascular system should therefore include palpation of the carotid, as already practised by many clinicians, and this can be supplemented when accurate timing is required by external carotid pulse recordings.

\section{SUMMARY}

External recordings of the carotid pulse have been compared in six patients with simultaneous recordings of the carotid pressure pulse: in the absence of hypertension, the external recordings satisfactorily reproduced the systolic part of the pressure pulse. External carotid recordings from 43 patients showed varying differences from the aortic pulse, but usually retained the same general form and were seldom inferior as a diagnostic guide. In the brachial pulse of 25 patients, however, many of the features of the central pulse were lost or greatly changed, so that the brachial pulse was of much less value in diagnosis than the external carotid.

I am grateful to Professor A. C. Dornhorst for his help in making the carotid pressure recordings. I would also like to thank Dr. A. Leatham for advice and criticism, and the Board of Governors of St. George's Hospital who provided a research grant.

\section{REFERENCES}

Daoud, G., Reppert, E. H., Jr., and Butterworth, J. S. (1959). Ann. intern. Med., 50, 323.

Duchosal, P. W., Ferrero, C., Leupin, A., and Urdaneta, E. (1956). Amer. Heart J., 51, 861.

Hancock, E. W., and Fleming, P. R. (1960). Quart. J. Med., 29, 209.

Kroeker, E. J., and Wood, E. H. (1955). Circulat. Res., 3, 623.

McDonald, D. A., and Taylor, M. G. (1959). In Prog. Biophys., Vol. 9, p. $105 . \quad$ Pergamon Press, London

Peterson, L. H., Jensen, R. E., and Parnell, J. (1960). Circulat. Res., 8, 622.

Robinson, B. (1963). Brit. Heart J., 25, 51.

Weissler, A. M., Peeler, R. G., and Roehll, W. H., Jr. (1961). Amer. Heart J., $62,367$.

Wright, J. L., and Wood, E. H. (1958). Amer. Heart J., 56, 64. 